\title{
A cloud computing scheduling and its evolutionary approaches
}

\author{
Ahmed Subhi Abdalkafor ${ }^{1}$, Alaa Abdalqahar Jihad ${ }^{2}$, Emad Tariq Allawi ${ }^{3}$ \\ ${ }^{1}$ Career Development Center, University Of Anbar, Anbar, Iraq \\ ${ }^{2}$ Computer center, University Of Anbar, Anbar, Iraq \\ ${ }^{3}$ Sunni Endowment, Anbar, Iraq
}

\begin{tabular}{l} 
Article Info \\
\hline Article history: \\
Received Nov 1, 2019 \\
Revised May 7, 2020 \\
Accepted Jul 12, 2020 \\
\hline
\end{tabular}

\section{Keywords:}

Cloud computing

Job scheduling

Load balancing

Task scheduling

\begin{abstract}
Despite the increasing use of cloud computing technology because it offers unique features to serve its customers perfectly, exploiting the full potential is very difficult due to the many problems and challenges. Therefore, scheduling resources are one of these challenges. Researchers are still finding it difficult to determine which of the scheduling algorithms are appropriate and effective and that helps increases the performance of the system to accomplish these tasks. This paper provides a broad and detailed examination of resource scheduling algorithms in the environment of a cloud computing environment and highlights the advantages and disadvantages of some algorithms to help researchers in selecting the best algorithms to schedule a particular workload to get a satisfy a quality of service, guarantee good utilization of the cloud resources also minimizing the make-span.
\end{abstract}

This is an open access article under the CC BY-SA license.

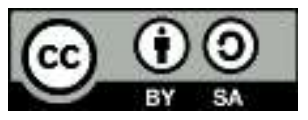

\section{Corresponding Author:}

Ahmed Subhi Abdalkafor

Career Development Center

University Of Anbar, Anbar, Iraq

Email: ahmed.abdalkafor@uoanbar.edu.iq

\section{INTRODUCTION}

The emergence of cloud computing has expanded dramatically and is becoming increasingly attractive as it provides services over the Internet and reduces user requirements for future planning [1,2]. The cloud has become mutually beneficial to both the service provider and the consumers. The cloud environment has many advantages that have made the world move strongly towards it. The cloud meets all the business requirements as well as improving the efficiency of managing resources through scheduling by algorithms and obtaining low cost and energy. However, the cloud environment has many challenges and difficulties, the system bugs and the power outages adversely affect the continuity of cloud computing working, Followed are some common examples of such problems: In the year 2007 Rackspace company stopped the service for 3 hours due to power outages from the company center. In 2008, the service of Google App Engine company was stopped for 6 hour due to errors in the operating system also in the year 2009 the company of Microsoft Azure discontinued 22 hours for updating an operating system [3].

In January 2018, an examination administered by the right scale company, 27\% of the respondents showed the shortage of resources or experience in this environment is a risk of cloud choosing for their works [4]. Figure 1 shows the cloud computing challenges in worldwide from 2018. The scheduling is an important and sensitive issue and has become an attractive topic in recent decades, in cloud computing environments that need to be carefully studied to make it easier for researchers to assign efficiently tasks to appropriate resources. Where it was considered as an NP-hard (nondeterministic polynomial time) problem due to large solution space and hence, it needs a big-time to get a better way solution [5], so scheduling methods are used to reach a high-quality service in a cloud environment. Therefore, you must meet the scheduling service 
requirements of different users. There will be many requests for service on the cloud platform at the same time. Therefore scheduling must be able to schedule multiple workflows [6].

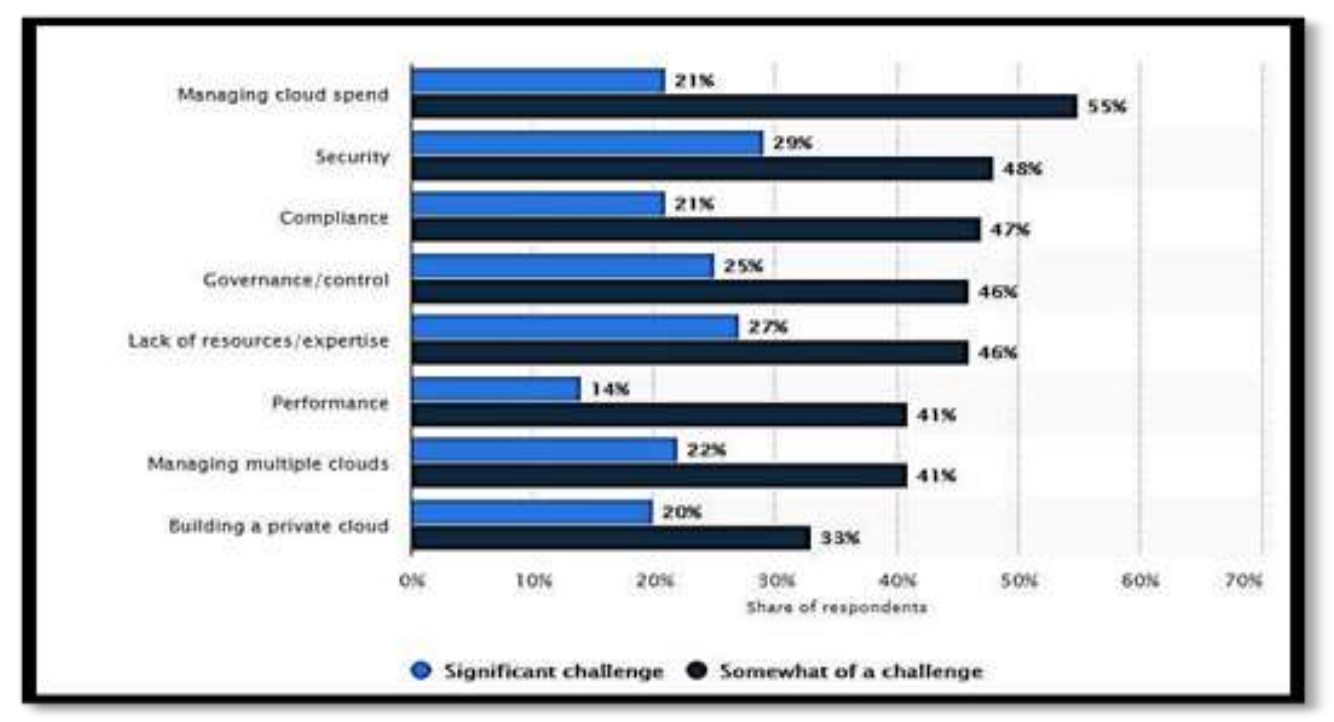

Figure 1. Cloud computing challenges from 2018

This paper aims to provide comprehensive and extensive an examination of scheduling algorithms in the environments of cloud computing where the highlight was in the key concepts of cloud scheduling, state-of-the-art implementation and architectural principles also investigated in the types of scheduling algorithms and a better understand the advantages and disadvantages of these algorithms.

The Scheduling is an NP-complete problem, by looking at a lot of problems such as keeping cost and energy down and reducing waiting time, therefore, many research methods have been proposed by many researchers. Despite the abundance of these studies on this hot topic, but the door is still open behind the researchers because most they reached are not perfect and not close to the optimal solution [7]. Below are some recent and important studies that will be used by researchers when researching in this field. Gibet T. and El Amrani [8] give a review of cloud computing infrastructure, discuss and evaluate its importance, and compute resources scheduling algorithms. Athokpam Bikramjit et.al [9] presented a comparative study and analyzed the several algorithms of scheduling and notes that the current algorithms provide big throughput and effective cost however, lack availability and reliability. S. Lakshmanan [10] presented a comparative analysis of ABC, FIFS, RR, SJF, Priority tasks, and merits, demerits scheduling algorithms in the cloud platform. These algorithms mainly focus on summarizing algorithm parameters, the job executes environment, types of job, it is used to decrease the make-span time, waiting for time and complexity of the code. Shameer A.P and A.C Subhajini [11] compared among three algorithms, round-robin (RR), Shortest job first (SJF) and first in first out (FIFO). Mohammad Masdari et.al [12] they offered the particle swarm optimization (PSO) algorithm based scheduling in an environment of a cloud computing, implemented a classification via the algorithm type (PSO), also presented how this algorithm was developed and combined to solve the problem of the task-workflow. Finally, a comprehensive comparison was provided via different objectives of scheduling like execution time, load balancing, and the costs.

Maria A. and Rajkumar B. [13] provide a study of the algorithms for scheduling scientific workflows. Focus on technologies considering applications formed as directed acyclic graph and the resource form given via providers of the public cloud. The classification is performed via a large study of being algorithms that focus on special features such as storage, network access, and VMs. Kamalpreet K. and Kanwalvir S. [14] they compared a variety of scheduling algorithms in tabular format various parameter making use of cloud computing environment in most of the algorithms. Simsy Xavier et.al [15] they have scanned several scheduling of workflow algorithms found in the environment of cloud then compared with different restrictions. Priya R. and Avinash P. [16] offers a survey of many scheduling of workflow algorithms kinds cloud computing, and it study compares several kinds of scheduling algorithms of a workflow. They coordinate problem of a scheduling via the environment of cloud and present a cloud scheduling hierarchy, principally divided to system and user levels. 


\subsection{Scheduling algorithm in cloud computing}

Kalka and Mohit [17] they adjusted the HEFT algorithm that inefficiently distributes the workload between the processor, reduces application rendering time based on their order and assignment of task with the processor. They concluded that the proposed algorithm decreases task time and load balance problems compared to the HEFT and CPOP algorithms. Ursa Sayeed et.al [18] proposed a hybrid FFA-PSO based AMTS method to reduce total energy consumption, total cost, implementation time, and increased resource operation. The result of the trial showed that the proposed FFA-PSO based AMTS works proficiently in a cloud computing in minimum implementation time, energy consumption and cost. J. KOK et.al [19] introduced by a new mechanism called modified max-min algorithm (MMax-Min), boosted by the Max-Min Algorithm. The suggested algorithm explores a cloud with maximum completion time and minimum completion time and assigns any of the clouds to implement according to specifications. They compared their proposal with standard max-min and round robin algorithms.

Mehdi Sookhak et.al [20] introduced an effective far data way audit structure to validate the integrity of the data kept via environment of cloud. They applied algebraic property data blocks that are outsourced to check file integrity remotely and decrease computational costs in their server and client from that environment. They planned structure of a new data that separates then subjugates the table for supportive effective data updating the bears minimal computing plus auditor of communications expenses. Arabi E. et.al [21] proposed the use of modified ant colony improvement in load balancing. This scheme enhances the scheduling of cloud jobs for load balancing. Amit A. and Saloni J. [22] the GPA focuses mainly on reducing mission time, this study virtual machines and tasks are ordered based on many instructions for each second. Results in the study were compared with tow algorithms first, Round Robin algorithm, and second first com first services, concluded, the GPA operates more useful than Round Robin and FCFS. M. Abu Sharkh et.al [23] introduce some internal and external factors affecting the design of DC RA models. Models operate based on a trusted social graph, and these models differ when compared to distributed cloud computing, grid, and classical computing models.

Ruay-Shiung Chang et.al [24] introduced the adaptive assessment function (ASJS) scheduling algorithm for the network environment. It proposes to shorten the end time, thus improving system productivity. This algorithm measures the computing power and transmission power for each cluster in the network system. Further, it measures the mass score for each block that will be adjusted by implementing local and global updates. Tasquia Mizan et.al [25] they introduced an improved the algorithm for tasking scheduling via a bee and a greedy algorithms for acquiring perfect service on hybrid cloud environment. Algorithms of Bee life are household to job scheduling with the greedy manner. In adjusted task scheduling, queue tasks are handled with a non-proactive priority queue. It enters the algorithm of BLA and greedy manner to acquire the perfect result. Machine size can be decreased with the hybrid method. Jiayin Li et.al [26] they proposed a scheduling algorithm based on the proposed comments for preventive functions in the cloud. The MinMin algorithm is used for scheduling jobs.

\section{TYPES OF SCHEDULING} scheduling.

Generally, Scheduling algorithms are divided into two main parts task scheduling and job

\subsection{Task scheduling}

Scheduling tasks are considered to be the most effective and efficient techniques for selecting the best resources in an efficient manner, a task scheduling classified into workflow, real-time scheduling, static and dynamic cloud service scheduling, and load balancing schedules.

a) Workflow: it is a manner that manages and maps the task execution on the assigned resources. It assigns appropriate resources to task of workflow in which the execution of the tasks can be finished to meet objective capacities needed by users. The workflow consists of from best-effort based scheduling algorithms and quality of service (QoS), the first one derived from a meta-heuristics based or heuristics-based approach. The second one means several applications of the workflow need some assurance of the quality of service. Therefore, these applications need to schedule so that they can analyze user service requirements.

b) Real-time scheduling: in general, the scheduling algorithm defines how tasks are handled by the scheduling system. In the scheduling system algorithm in real-time each description of the task and the deadline and the identifier are set and completed As defined according to scheduling algorithms, Scheduling algorithms must prevent concurrent way to devices and shared resources. The problem of scheduling of the real-time system is to set a schedule to perform tasks so that its timing constraints are met. In simpler real-time systems where tasks and execution times are known to all may not even be a scheduler simply perform a task (or give in to) the next. In the most complex real-time system with a larger (but static) number, may be possible to work a fixed schedule based on the required run tasks and 
the time expected to be completed for each list where ensures the implementation of all tasks in a timely manner. But for many systems in real-time the workload changes from moment to moment depending on external events this requires dynamic scheduling. The system is said to be un-schedulable when tasks cannot meet deadlines, the task can be classified as a periodic or non-periodic process [27].

c) Static and dynamic cloud service scheduling: there are two scheduling tasks in the cloud; Schedule static tasks and schedule dynamic tasks. Static scheduling allows you to fetch the required data and connect all the scheduling parameters initially scheduling a static schedule imposes a lower load at runtime. For dynamic scheduling, the task/task modules information is unknown. Therefore, the task of execution time may not be known and tasks are allocated quickly. The advantage of a dynamic static schedule that the system does not have to possess the run-time conduct of the request previously running it. An additional the dynamic scheduler is divided into a couple of types batch mode or connected mode. In first one, the tasks are queued, grouped in a group, and then scheduling after time interval. In second, the tasks are scheduled after they access the system [28, 29].

d) Load balancing: it is one of the best significant concepts in distributed environments. A Cloud computing is also one of the better platforms that availability data storage at the lowest cost and can be accessed at any time online. The basic feature of the load balancer is to improve resource utilization, maximize productivity, reduce response time, and avert overcharging of resources [30]. The aim of load balancing to the high level of user satisfaction and the use of the proportion of resources by ensuring a reasonable and well done for each resource allocation of computing resources. The load balancing application reduces the opportunity for interruptions that can simultaneously affect the services of cloud systems. If a single system interruption is affected, the load balancer is capable of switching to another available resource. Additionally, confirms that every processor or each node does almost equal work at any time. There are various types of load balancing algorithms available, which can be categorized mainly into two groups, static algorithms and dynamic algorithms.

\subsection{Job scheduling algorithms}

At present, cloud computing is used widely. hence, several challenges in the use of cloud computing. The resource management challenge and one of the resource-related administration issues is the scheduling of tasks [31]. Scheduling work is to achieve excellent performance computing and the most useful productivity system. The principal task of scheduling algorithms in the cloud reduces the total time to complete the tasks by obtaining the most suitable resources that will be customized to the task. The environment is a various system and the activity of each processor varies rapidly, and the priority of the job is an important issue in scheduling for some of the functions services must be earlier than those other functions cannot be kept for long in this system. You should take the appropriate scheduling functions into account for the priority functions algorithm [30]. In general, the job Scheduling algorithms is consists of two main parts, the first parts are Batch-modeheuristic-scheduling-algorithms which consists of several algorithms such as round robin algorithm, randomized, max-min scheduling algorithm min-min algorithm, shortest job first algorithm, and others and online-mode-heuristic-algorithms in the second part that mean when a task is arrived directly mapped onto a host so this technique is more appropriate because the heterogeneous and speed of each processor changes fast in this environment. The appropriate example for this part is the most fit task scheduling [32].

a) The round robin algorithm: specially designed for time-sharing systems. The load is distributed equally to all resources in circular order. Run a slide function (sometimes called a quantum scheduler). The first operation of the queue is selected from the CPU scheduler and then sets the timer to Cut off after quantum one the finally sending the process. Do this repeatedly until the jobs are completed. If the job does not fulfill in a single custom quantity, revert to the queue and wait for the next round. The Jobs are performed in a consecutive cycle, and a necessity to wait for the earlier job to get full. Consequently, there is no famine problem [33].

b) Randomized algorithm: is the simplest algorithm available that uses a degree of randomness as part of its logic? Two advantages of random distribution have simplicity and speed; this effectively reduces the complexity of the account. The algorithm typically uses a random bit uniformly as a helper input to direct its behavior. The performance of the algorithm will be a random variable defined by random bits, thus either time management, or output (or both) random variables.

c) Max-min scheduling algorithm: it chooses big tasks to be implemented first. Here, we are given the greater priority tasks on the smaller tasks, if we have one long job, Max-Min can perform several short tasks simultaneously while Implementation a large one. Continue to work in descending order. Which again repeats this procedure for all available functions [34-36].

d) Min-min algorithm: this algorithm determines the less expected time of tasks to be executed. This algorithm passes through two stages, the first is to search for the less time expected to accomplish all the tasks. Then the second step is to assign the specific task that was performed in the first step depending on the overall less 
expected completion time to the correct resource [37, 38]. Regardless of the advantages that exist in this algorithm of obtaining the least time where there is starvation for some of the tasks when executed.

e) Shortest job first algorithm: there are many algorithms available to schedule the CPU including the first shorter function (SJF). A high priority is allocated to posts with minimum execution time and put first in the queue while the lowest priority is allocated to the task with maximum implementation time, always select the shortest function (any job takes less time) available. When the CPU is available [32]. Table 1 illustrated the advantage and disadvantage of algorithms that which were mentioned and Figure 2 shows the types of scheduling algorithms.

On the other hand, the pattern recognition is an important and fertile topic as it has entered many applications such as recognizing the different languages from Arabic, English, and other languages of the world [39], so, many databases have been built to implement and test these applications [40-44], as result, the recognition has entered a large area in many applications in the cloud environment such as Emotion of human [45] and activity recognition [46], and face recognition [47-49].

Table 1. Pros and cons of scheduling algorithms

\begin{tabular}{|c|c|c|}
\hline Name of Algorithms & Advantage & Disadvantage \\
\hline $\begin{array}{l}\text { Round Robin } \\
\text { Algorithm }\end{array}$ & $\begin{array}{l}\text { Easier for implementing, and it is free from } \\
\text { famine (which means that the process does } \\
\text { not have the resources to complete it } \\
\text { permanently or after a long time. and tasks } \\
\text { processing without priority }\end{array}$ & $\begin{array}{l}\text { The average waiting time is worse than FCFS, } \\
\text { and then the performance level depends on the } \\
\text { slice. }\end{array}$ \\
\hline $\begin{array}{l}\text { Randomized } \\
\text { Algorithm }\end{array}$ & Simplicity, Performance & $\begin{array}{l}\text {-A run-time analysis or a suitable solution is } \\
\text { often complex. } \\
\text {-Improbable for Producing random number. } \\
\text { The result depends a large part on the quality of } \\
\text { the random numbers, so it needs to rely on false } \\
\text { random numbers }\end{array}$ \\
\hline $\begin{array}{l}\text { Max-Min scheduling } \\
\text { algorithm }\end{array}$ & $\begin{array}{l}\text { Performance level depends on tasks if the } \\
\text { task is longer. }\end{array}$ & $\begin{array}{l}\text { Priority depends on less important. They are } \\
\text { positive on the basis of a big task more than a } \\
\text { small task }\end{array}$ \\
\hline Min-Min Algorithm & Obtaining least time & $\begin{array}{c}\text { Starvation for some of the tasks when executed } \\
\text {-Difficulty predicting CPU time and } \\
\text { implementation. }\end{array}$ \\
\hline $\begin{array}{l}\text { Shortest Job First } \\
\text { Algorithm }\end{array}$ & $\begin{array}{l}\text { Make waiting time for operations less } \\
\text { executable. }\end{array}$ & $\begin{array}{l}\text {-In the event that the waiting period is } \\
\text { shortened, as long-term operations may wait for } \\
\text { a long time and may not be carried out, so they } \\
\text { have a problem, (starvation of large process) } \\
\text { famine of long-term processes. }\end{array}$ \\
\hline
\end{tabular}

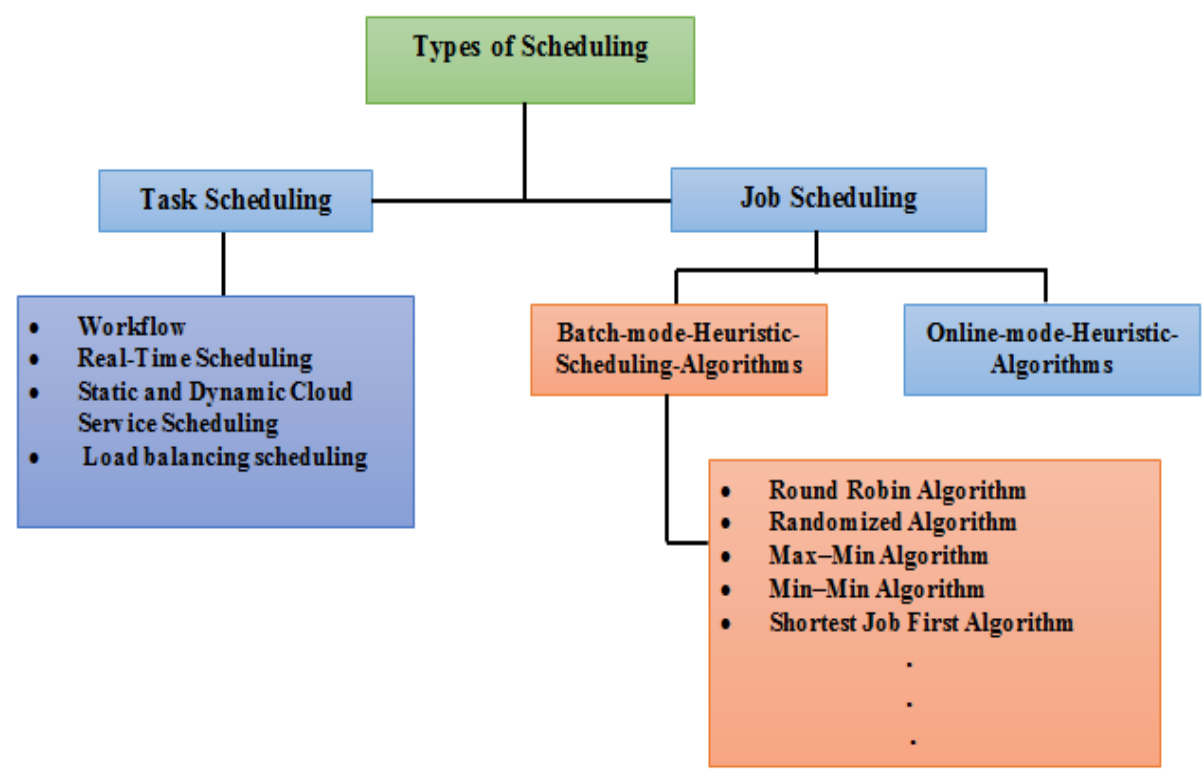

Figure 2. The types of scheduling algorithms 


\section{CONCLUSION}

The scheduling is one of the most important matters which need to be focused to obtain efficient performance in cloud computing. In this research, we conducted a broad, comprehensive, and intensive examination of the scheduling of the algorithms as well as focus on the most important studies in this field. We also discussed the advantages and disadvantages of some scheduling algorithms to be selecting the best algorithms by researchers to achieve utilization of the resource, completion cost and time according to the comprehensive examination of these algorithms and also the pros and cons of these algorithms.

\section{REFERENCES}

[1] Isa, A. W. M, et al., "Cloud computing adoption reference model," Indonesian Journal of Electrical Engineering and Computer Science, vol. 16, no. 1, pp. 395-400, October 2019.

[2] Olanrewaju, R. F., Islam, T., Khalifa, O. O., \& Fajingbesi, F. E., "Data in Transit Validation for Cloud Computing Using Cloud-Based Algorithm Detection of Injected Objects," Indonesian Journal of Electrical Engineering and Computer Science, vol. 10, no. 1, pp. 348-353, 2018.

[3] Qian, L., Luo, Z., Du, Y., \& Guo, L., "Cloud computing: An overview," In IEEE International Conference on Cloud Computing, pp. 626-631, 2009.

[4] https://www.statista.com/statistics/511283/worldwide-survey-cloud-computing-risks/accessed at 24/5/2019.

[5] Chang, Ruay-Shiung, Chih-Yuan Lin, and Chun-Fu Lin, "An adaptive scoring job scheduling algorithm for grid computing," Information Sciences, vol. 207, pp. 79-89, 2012.

[6] Zhou, Y., \& Huang, X., "Scheduling workflow in cloud computing based on ant colony optimization algorithm," In Business Intelligence and Financial Engineering (BIFE), 2013 Sixth International Conference on IEEE, pp. 57-61, 2013.

[7] Choudhary, A., Gupta, I., Singh, V. \& Jana, P. K., "A GSA based hybrid algorithm for bi-objective workflow scheduling in cloud computing," Future Generation Computer Systems, vol. 83, pp. 14-26, 2018.

[8] Gibet Tani Hicham, El Amrani Chaker, "Optimization of Task Scheduling Algorithms for Cloud Computing: A Review," SCAMS-SH'17, October 2017.

[9] Athokpam Bikramjit Singh, Sathyendra Bhat J., Ragesh Raju, Rio D’Souza, "A Comparative Study of Various Scheduling Algorithms in Cloud Computing,” American Journal of Intelligent Systems, vol. 7, no. 3, pp. 68-72, 2017.

[10] S. Lakshmanan, "A Survey on Heterogeneous Resource Scheduling Algorithm in Cloud Computing," IJSRSET, vol. 3, no. 8, pp. 962-966, 2017.

[11] Shameer A. P., "Study on Different Scheduling Algorithm for Cloud Computing," International Journal of Advanced Research in Computer Science and Software Engineering, volume 6, issue 5, May 2016.

[12] M. Masdari, F. Salehi, M. Jalali, and M. Bidaki, "A Survey of PSO-Based Scheduling Algorithms in Cloud Computing," J. Netw. Syst. Manag., vol. 25, no. 1, pp. 122-158, Jan. 2016.

[13] Maria A. Rodriguez and Rajkumar Buyya, A Taxonomy and Survey on Scheduling Algorithms for Scientific Workflows in IaaS Cloud Computing Environments, Concurrency and Computation: Practice and Experience (CCPE), volume 29, no. 8, pp. 1-23, ISSN: 1532-0626, Wiley Press, New York, USA, April 25, 2017

[14] Kamalpreet Kaur, Kanwalvir Singh Dhindsa, "Comparative Study of Tools and Scheduling Algorithms of Cloud Computing," International Conference on Communication, Information and Computing Technology, 2015.

[15] Simsy Xavier, S.P.Jeno Lovesum, "A Survey of Various Workflow Scheduling Algorithms in Cloud Environment," International Journal of Scientific and Research Publications, vol. 3, no. 2, 2013.

[16] Priya R. Lodha and Avinash P. Wadhe, "Study of Different Types of Workflow Scheduling Algorithm in Cloud Computing," IJARCSEE, vol. 2, no. 4, April 2013.

[17] Kalka Dubey, Mohit Kumar, S.C. Sharma, "Modified HEFT Algorithm for Task Scheduling in Cloud Environment," Procedia Computer Science, vol. 125, pp. 725-732, 2018.

[18] Ursa Sayeed, Arshad Shareef, T Sunil, "An Optimal Task Scheduling Strategy in Cloud Computing Environment Utilizing FFA-PSO Algorithms," International Journal of Advance Research in Science \& Engineering, 2017.

[19] J. Kok Konjaang, Fahrul Hakim Ayob, Abdullah Muhammed, "An Optimized Max-Min Scheduling Algorithm in Cloud Computing," Journal of Theoretical and Applied Information Technology, vol. 95, no. 9, 2017.

[20] Mehdi Sookhak, Abdullah Gani, Muhammad Khurram Khan, Rajkumar Buyya, "Dynamic remote data auditing for securing big data storage in cloud computing," Information Scienses, 2015.

[21] Arabi E. Keshk, Ashraf B. El-Sisi, Medhat A. Tawfeek, "Cloud Task Scheduling for Load Balancing based on Intelligent Strategy," I. J. Intelligent Systems and Applications, vol. 6, no. 5, pp. 25-36, 2014.

[22] Amit Agarwal, Saloni Jain, "Efficient Optimal Algorithm of Task Scheduling in Cloud Computing Environment," International Journal of Computer Trends and Technology, vol. 9, pp. 344-349, 2014.

[23] M. Abu Sharkh, M. Jammal, A. Shami and A. Ouda, "Resource allocation in a network-based cloud computing environment: design challenges," in IEEE Communications Magazine, vol. 51, no. 11, pp. 46-52, November 2013. doi: 10.1109/MCOM.2013.6658651.

[24] Chang, Ruay-Shiung, Chih-Yuan Lin, and Chun-Fu Lin, "An adaptive scoring job scheduling algorithm for grid computing," Information Sciences, vol. 207, pp. 79-89, 2012.

[25] Mizan, T., Masud, S. M. R. A., Latip, R., "Modified Bees Life Algorithm for Job Scheduling in Hybrid Cloud," International Journal of Engineering and Technology, vol. 2, no. 6, pp. 974-979, June, 2012.

[26] Li, M. Qiu, J. Niu, W. Gao, Z. Zong and X. Qin, "Feedback Dynamic Algorithms for Preemptable Job Scheduling in Cloud Systems," 2010 IEEE/WIC/ACM International Conference on Web Intelligence and Intelligent Agent Technology, Toronto, ON, pp. 561-564, 2010. doi: 10.1109/WI-IAT.2010.30. 
[27] S. Liu, G. Quan and S. Ren, "On-Line Scheduling of Real-Time Services for Cloud Computing,” 2010 6th World Congress on Services, Miami, FL., pp. 459-464, 2010. doi: 10.1109/SERVICES.2010.109.

[28] Akilandeswari, P., \& Srimathi, H., "Survey and analysis on Task scheduling in Cloud environment," Indian Journal of Science and Technology, vol. 9, no. 37, pp. 1-6, 2016.

[29] Goel, H., \& Chamoli, N., "Job Scheduling Algorithms in Cloud Computing: A Survey," International Journal of Computer Applications, vol. 95, no. 23, pp. 19-22, 2014.

[30] Athokpam Bikramjit Singh, Sathyendra Bhat J., Ragesh Raju, Rio D’Souza, "A Comparative Study of Various Scheduling Algorithms in Cloud Computing,” American Journal of Intelligent Systems, vol. 7, no. 3, pp. 68-72, 2017.

[31] Hamad, S. A., \& Omara, F. A., "Genetic-based task scheduling algorithm in cloud computing environment. International Journal of Advanced Computer Science and Applications, vol. 7, no. 4, pp. 550-556, 2016.

[32] Pandaba Pradhan, Prafulla Ku. Behera, B. N. B. Ray, "Modified Round Robin Algorithm for Resource Allocation in Cloud Computing," Procedia Computer Science, vol. 85, pp. 878-890, 2016.

[33] Abdalkafor, A. S., \& Alheeti, K. M. A., "A hybrid approach for scheduling applications in cloud computing environment," International Journal of Electrical and Computer Engineering (IJECE), vol. 9, no. 4, 2019.

[34] Rawshdeh, D. Zanoon, "STASR A New Task Scheduling Algorithm For Cloud Environment," Network Protocols and Algorithms, vol. 7, no. 2, pp. 81-95, 2015.

[35] El Amrani, C., \& Gibet Tani, H., "Smarter Round Robin Scheduling Algorithm for Cloud Computing and Big Data," Journal of Data Mining \& Digital Humanities, vol. 7, no. 2, pp. 1943-3581, 2018.

[36] Athokpam Bikramjit Singh, Sathyendra Bhat J., Ragesh Raju, Rio D’Souza, "A Comparative Study of Various Scheduling Algorithms in Cloud Computing,” American Journal of Intelligent Systems, vol. 7, no. 3, pp. 68-72, 2017.

[37] Kaur, K., Kaur, N., \& Kaur, K., "A Novel Context and Load-Aware Family Genetic Algorithm Based Task Scheduling in Cloud Computing," In Data Engineering and Intelligent Computing, Springer, pp. 521-531, 2017.

[38] Manasrah, A. M., \& Ba Ali, H., "Workflow scheduling using hybrid GA-PSO algorithm in cloud computing," Wireless Communications and Mobile Computing, vol. 3, pp. 1-16, 2018.

[39] S. Abdalkafor, "Designing Offline Arabic Handwritten Isolated Character Recognition System using Artificial Neural Network Approach,” International Journal Of Technology, vol. 8, pp. 528-538, January 2017.

[40] S. Abdalkafor, "DFRS-database for fingerprint recognition system using Ink-On-Paper technique," Journal of Engineering and Applied Sciences, vol. 13, no. 17, pp. 7401-7407.

[41] A. S. Abdalkafor, E. T. Allawi, K. W. Al-Ani and A. M. Nassar, "A Novel Database for Arabic Handwritten Recognition (NDAHR) System," 2019 2nd International Conference on Computer Applications \& Information Security (ICCAIS), Riyadh, Saudi Arabia, pp. 1-6, 2019. doi: 10.1109/CAIS.2019.8769580.

[42] Abdalkafor, A. S., "Survey for Databases On Arabic Off-line Handwritten Characters Recognition System," In 2018 1st International Conference on Computer Applications \& Information Security (ICCAIS), pp. 1-6, April 2018,

[43] Abdalkafor, A. S., Awad, W. K., \& Alheeti, K. M. A., "A novel comprehensive database for Arabic and English off-line handwritten digits recognition," Indonesian Journal of Electrical Engineering and Computer Science, vol. 20, no. 1, pp. 145-149, 2020.

[44] Jihad, A. A., \& Abdalkafor, A. S. (2019). A Framework for Sentiment Analysis in Arabic Text. Indonesian Journal of Electrical Engineering and Computer Science, 16(3), 1482-1489.

[45] Lu, H., Wang, M., \& Sangaiah, A. K., "Human Emotion Recognition Using an EEG Cloud Computing Platform," Mobile Networks and Applications, vol. 25, no. 3, pp. 1023-1032, 2020.

[46] Islam, N., Faheem, Y., Din, I. U., Talha, M., Guizani, M., \& Khalil, M., "A blockchain-based fog computing framework for activity recognition as an application to e-Healthcare services," Future Generation Computer Systems, vol. 100, pp. 569-578, 2019.

[47] T. Soyata, R. Muraleedharan, C. Funai, M. Kwon and W. Heinzelman, "Cloud-Vision: Real-time face recognition using a mobile-cloudlet-cloud acceleration architecture," 2012 IEEE Symposium on Computers and Communications (ISCC), Cappadocia, pp. 000059-000066, 2012. doi: 10.1109/ISCC.2012.6249269.

[48] M. Z. Khan, S. Harous, S. U. Hassan, M. U. Ghani Khan, R. Iqbal and S. Mumtaz, "Deep Unified Model For Face Recognition Based on Convolution Neural Network and Edge Computing," in IEEE Access, vol. 7, pp. 7262272633, 2019. doi: 10.1109/ACCESS.2019.2918275.

[49] Guo, S., Xiang, T., \& Li, X., "Towards efficient privacy-preserving face recognition in the cloud. Signal Processing," vol. 164, pp. 320-328, 2019.

\section{BIOGRAPHIES OF AUTHORS}

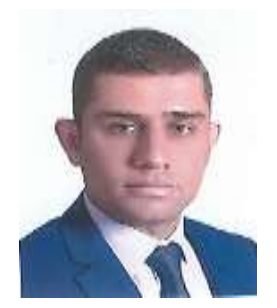

Ahmed Subhi Abdalkafor was born in Anbar-Iraq in 1988. He received his B.Sc. from Faculty of Computer Science at Anbar University, Iraq in 2010. The MSc. degree from Computer Science Department in Middle East University, Jordan in 2016. His research interests are, Image Processing, Pattern Classification, Artificial Intelligent, Wireless Sensor Neural Network, Cloud Computing, Machine Learning and Data Mining. 

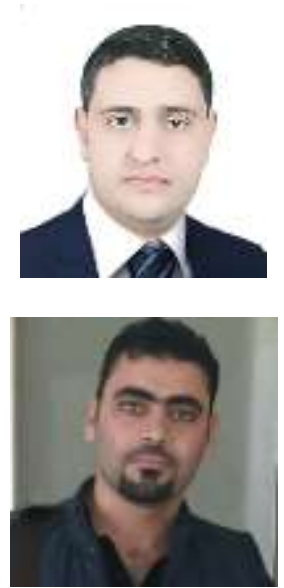

Alaa Abdalqahar Jihad was born in Anbar-Iraq in 1985. He received his B.Sc. from Faculty of Computer Science at Anbar University, Iraq in 2009. The MSc. degree Faculty of Computer Science at Anbar University, Iraq 2012. His research interests are, Data Warehouse, Data Mining, Artificial Intelligent, Machine Learning, Cloud Computing and Natural Language Processing

Emad Tariq Allawi was born in Anbar-Iraq in 1982. He received his B.Sc. from Faculty of Computer Science at Almaref University, Iraq in 2006. The MSc. degree from Computer Science Department in Middle East University, Jordan in 2016. His research interests are Data Mining, Image Processing and cyber Security. 